\title{
DEFINICIÓN DE LA POLÍTICA PÚBLICA EN LA PREVENCIÓN Y MANEJO DEL MALTRATO Y EL ABUSO: BIOÉTICA HERMENÉU- TICA Y SALUD PÚBLICA COMO REFERENTES
}

\author{
Carmen Alicia Cardozo de Martínez¹, Olga del Carmen Maldonado Gómez², Alicia García González ${ }^{2}$ \\ y Galo Segovia Baus, S.J. ${ }^{2}$
}

Resumen: La bioética hermenéutica, como práctica racional, razonada y sensitiva, permite poner en movimiento, a través de la deliberación, el diálogo y el análisis interpretativo, diferentes respuestas discursivas que contribuyen a dar un nuevo significado a las condiciones de las relaciones intersubjetivas sociales y familiares, en el marco tanto de la intencionalidad ética, orientada a la vida buena (teleológico), como en el marco normativo (deontológico), como complementarios. En el maltrato y abuso infantiles es necesario generar nuevas comprensiones y discursos, como resultado de las nuevas configuraciones sociales, culturales y filiales, que simultáneamente superen la dicotomía persistente víctima-victimario y que permitan interpretar, reinterpretar y proponer nuevos planteamientos para la política pública y la salud pública. Este artículo evidencia la posibilidad de generar nuevos enfoques integradores —éticos, políticos y sociales_, sobre la manera como se comprende el maltrato infantil, en una perspectiva compleja y dinámica, que lo sitúa en el campo de las interacciones humanas y de sus vicisitudes.

Palabras clave: bioética hermenéutica, salud pública, política pública, categorías de encuentro, maltrato y abuso infantil

Definition of public policy in the prevention and management of maltreatment and abuse: hermeneutics of bioethics and public health as relevant

\begin{abstract}
Bioethics hermeneutics as rational practice, reasoned and sensitive, can set in motion, through deliberation, dialogue and interpretive analysis, different discursive responses that contribute to give new meaning to the conditions of social inter- relationships and family constructions under both the ethical aim, good life oriented (teleological) and in the regulatory framework (ethics). In abuse and child abuse is necessary to generate new understandings and discourses as a result of the new social , cultural and affiliates forms; simultaneously overcome the dichotomy persistent offender and victim to interpret, reinterpret and propose new approaches to public policy and public health. This paper demonstrates the possibility of generating new elements from the leaders in integrative approaches, ethical, political and social shifts in the way where child abuse is understood in a complex and dynamic perspective, which places it in the field of human interactions and its vicissitudes, It is pretended to construct mutual relationships and consideration for the other, based on the perspective of social bioethics and hermeneutics , to create spaces for coordination with public health, in which " the intentionality of the good life " with and in just institutions" are the axes.
\end{abstract}

Key words: bioethics hermeneutics, public health, public policy, meeting categories, abuse and child abuse

Definição da política pública na prevençáo e manejo do maltrato e abuso: bioética hermenêutica e saúde pública como referências

Resumo: A bioética hermenêutica, como prática racional, fundamentada e sensitiva, permite por em movimento, por meio da deliberação, do diálogo e da análise interpretativa, diferentes respostas discursivas que contribuem para dar um novo significado às condiçôes das relaçóes intersubjetivas sociais e familiares, no marco tanto da intencionalidade ética, orientada para a vida boa (teleológico), como no marco normativo (deontológico), como complementares. No maltrato e abuso infantis é necessário gerar novas compreensóes e discursos, como resultado das novas configuraçôes sociais, culturais e filiais, que simultaneamente superem a dicotomía persistente vítima-vitimário e que permitam interpretar, reinterpretar e propor novas proposiçóes para a política pública e saúde pública. Este artigo evidencia a possibilidade de gerar novos enfoques integradores —éticos, políticos e sociais - sobre a maneira como se compreende o maltrato infantil, numa perspectiva complexa e dinâmica, que o situa no campo das interaçôes humanas e de suas vicissitudes.

Palavras-chave: bioética hermenêutica, saúde pública, política pública, categorias de encontro, maltrato e abuso infantil

\footnotetext{
${ }^{1}$ Departamento de Ciencias Básicas, Facultad de Odontología, Universidad Nacional de Colombia, Colombia Correspondencia: carmen_aliciademartinez@yahoo.co.uk

${ }^{2}$ Maestría en Bioética, Pontificia Universidad Javeriana, Colombia
} 


\section{Introducción}

La complejidad situacional, contextual y sociohistórica en la prevención y manejo del maltrato y abuso infantiles, ha llevado a la generación y propuesta constante de modelos que atenúen, disminuyan o modifiquen esta forma de relaciones interhumanas, estudiadas y clasificadas desde distintas perspectivas, definiendo asimismo cursos de acción que permitan o promuevan la protección de los actores involucrados directa o indirectamente en estos procesos.

La racionalidad imperante por tradición ha sido la jurídica, lo que ha permitido establecer vías de solución no siempre ideales, justas o eficaces, pero que tienen un soporte claro, contundente, y un ordenamiento que permite argumentaciones a favor y en contra, soportadas en esta misma lógica, y que pueden llevar, en algunas ocasiones, a buenas alternativas, pero en su mayoría a grandes frustraciones. Estas determinaciones de carácter legal tienen su soporte en lo establecido en la denominada "política pública", la cual igualmente tiene su propia lógica y racionalidad.

Sin embargo, los resultados y las evidencias muestran que las unas y las otras presentan grandes vacíos, debilidades y en muchas ocasiones efectos indeseables sobre las personas y las familias(1). Lo que tienen todas en común es la evidencia de haber excluido a los verdaderos protagonistas que enfrentan los conflictos y deben dar soluciones, pues no existen herramientas o claros mecanismos que permitan incluirlos en los círculos de decisión.

La legislación nacional colombiana establece que los casos de abuso y maltrato deben ser orientados, guiados y decididos por autoridades competentes, como se establece en la Ley 1.098 de 2006, Código de la Infancia y la Adolescencia, artículos 79 a 95. Si bien es cierto que las personas encargadas de estos procesos tienen conocimiento, experiencia, soporte administrativo y, en algunas ocasiones, acierto en sus acciones, la práctica evidencia una serie de factores preocupantes con relación a la perpetuación de las conductas que lesionan la condición de niños, niñas, adolescentes, hombres y mujeres, entre los cuales se destacan dos aspectos: que no es suficiente la determinación normativa y que las políticas públicas presentan una serie de vacíos, desaciertos o interpretaciones que las hacen ineficientes, insuficientes y algunas veces inviables. Finalmente, las dinámicas de análisis colectivo, como las de la salud pública, al parecer no se insertan de manera armónica en la cotidianidad normativa, precisamente por tener otra lógica en su conformación y aplicación, basada fundamentalmente en conceptos de carácter epidemiológico nosológico.

Específicamente, más allá de la norma y sus procedimientos, se encuentran las experiencias, vivencias e interpretaciones de quienes aplican las determinaciones de tipo legal. Seres humanos que tienen diversas visiones de la vida, de las interacciones personales, de la constitución de la familia, de la sociedad, del ser hombre, mujer, niño, niña, adolescente, anciano o anciana; que tienen diversos conceptos sobre responsabilidad, culpabilidad y roles, y que asimismo tienen diversas maneras de construir la valoración moral de sus actos y relaciones con los demás.

Esto quiere decir que, aunque el funcionario responsable de aplicar la norma entienda el sentido de una regulación que está en obligación de ejecutar, pesa más en su decisión su compromiso moral, su sentido del deber según sus creencias, principios y valores que lo que pueda decir la norma per se. Puede mencionarse aquí el tan conocido caso de la "objeción de conciencia" frente a la interrupción voluntaria del embarazo, en el que la norma reconoce los tres casos que en Colombia se despenalizan y exceptúan para que la mujer pueda acceder a un procedimiento seguro $y$, sin embargo, tanto médicos como abogados, por sus creencias, buscan la manera de impedir a la mujer ejercer este derecho, por prevalecer, en general, el concepto patriarcal en la decisión(2).

La reinterpretación y la búsqueda de sentido entre las diferentes racionalidades y sensibilidades personales e interpersonales, mediante el diálogo ciudadano libertario, constituiría un espacio analítico ideal para la definición de mejores cursos de acción en problemas complejos colectivos, situación que podría ser resuelta en principio a través de la bioética hermenéutica, por lo que podría contribuir no solo a la interpretación del contexto del abuso y el maltrato, sino a prevenir la per- 
petuación de esta conducta, dando paso así a un posible cambio en su abordaje y manejo social.

Entendemos la bioética como el uso creativo del diálogo ciudadano para promover la deliberación interdisciplinaria e intersectorial, en condición participativa plural, para la búsqueda conjunta de la mejor solución en el estudio o análisis de problemas o conflictos complejos. También permite lograr la disminución de los conflictos que se generan en el devenir humano. En nuestro caso la constituyó la bioética social o bioética hermenéutica, como modelo de interpretación, expresión y transformación.

Esta bioética hermenéutica es práctica y aplicada y aplicable a los contextos sociales en los que se estudian aquellos aspectos que se encuentran más allá de las políticas públicas, de las normas legales y del contexto asistencial.

La salud pública es entendida como la intervención colectiva, tanto del Estado como de la sociedad civil, orientada a proteger y mejorar la salud de las personas(3), desde la perspectiva del análisis de factores asociados a grupos o comunidades, en contraposición con el concepto asistencial individual de atención médica, que promueve la salud de las personas. La salud pública es un saber hacer distinto al de la relación médico-paciente y contempla interpretaciones comportamentales, sociales o comunitarias.

En este artículo se pretende aproximar la bioética y la salud pública en el ejercicio ponderativo valórico para la definición de la política pública en maltrato y abuso infantil, utilizando la bioética hermenéutica.

\section{Antecedentes}

La bioética, como campo del conocimiento en construcción, ha mostrado en varios escenarios que puede ser una salida adecuada a la solución o al menos disminución de los conflictos sociales en los cuales están involucradas las consideraciones morales de las personas, por lo que nuestro grupo de trabajo consideró que, con esta nueva perspectiva, podríamos avanzar en la generación de un nuevo modelo para el diseño y la ejecución de la política pública en abuso y maltrato infantil en Colombia. Aproximar conceptos como salud y política pública en los escenarios cotidianos de la aplicación normativa vigente en abuso y maltrato, partiendo de una categoría nueva dada por la bioética —el reconocimiento o inclusión, mi conciencia en relación con el otro- en escenarios de diálogo que busquen justicia, podría ser una respuesta que facilite el avance en la superación de los conflictos propios de la violencia intrafamiliar en Colombia y otros países latinoamericanos.

\section{Perspectivas de la bioética y la salud pública}

La bioética y la salud pública tienen en común el estudio del comportamiento humano y de sus interrelaciones desde diferentes perspectivas: la primera tiene como sustrato el referente axiológico y la segunda los indicadores de la llamada "dimensión colectiva de la salud", representada principalmente por la ausencia de enfermedad, de lesiones y de incapacidad, en un completo estado de bienestar. Aunque los dos conceptos son aplicables a campos específicos del conocimiento y pueden contar con su propio estatuto epistemológico, hay una gran controversia, en especial en lo relacionado con la definición, alcances y perspectivas de la bioética.

La bioética, que empieza reflexionando sobre aspectos relacionados con el ejercicio profesional y los conflictos asistenciales en los comienzos y finales de la vida, se va transformando hasta alcanzar niveles de elaboración conceptual más en el contexto que en la búsqueda del sentido mismo de la vida(4), del trasfondo del valor de las relaciones interhumanas, y de conceptos tan importantes para la definición de acciones prácticas en la cotidianidad ciudadana como lo público y lo privado(5). Se establecen entonces nuevos horizontes que demarcan principios distintos a aquellos inicialmente esbozados, aplicados y repetidos en muchos artículos, y denominados por algunos como el "mantra de Georgetown" (beneficencia, no maleficencia y justicia), orientados indudablemente a la aplicación de acciones en la atención individual.

Según propone la Unesco, la dignidad humana, la libertad, los derechos humanos y la justicia se incluyen en el respeto a la diversidad cultural y el pluralismo, con los principios de solidaridad, 
equidad, cooperación y responsabilidad con la naturaleza. Por esto, afirma Hoyos, se consideran principios derivados de los anteriores el de la primacía de la persona, la no discriminación, la autonomía y la responsabilidad, el consentimiento informado, la privacidad y la confidencialidad, así como la obligación de compartir equitativamente los beneficios de la investigación. Por lo anterior, se propone situar estos planteamientos de la bioética en un punto de partida desde la filosofía moral, permitiendo comprenderla como un campo especial de articulación de la moral. Lo cual implica la realización de un ejercicio reflexivo deliberativo, con profundidad argumentativa, que promueva una postura plural y que represente las diversas formas de pensamiento y los diversos cursos de acción frente a una situación conflictiva.

La esencia entonces es la búsqueda de la fundamentación moral de los actos humanos en todos los escenarios posibles de la vida, entre individuos o entre colectivos o grupos humanos, para la construcción de una verdadera ciudadanía, entendida esta como la capacidad cívica o civil de comprender al otro en todas sus dimensiones, sin ningún tipo de sesgo.

Esto significa entonces que una bioética social(6), una bioética pública(7) o una bioética hermenéutica(8) podrían constituir conceptualmente la base para el análisis reflexivo sensitivo de la política pública y su ejecución en maltrato y abuso infantil.

Este diálogo reflexivo tiene como características: el análisis crítico plural de los problemas abordados en un estudio interdisciplinario e intersectorial de carácter ciudadano, en el que participan técnicos expertos y no expertos en el tema, pero que tienen una percepción social o sensible de estos problemas; la propuesta de cursos de acción posibles como escenarios fácticos proyectados por la experiencia y conocimiento de los propios actores; la horizontalidad en las relaciones intersubjetivas de los participantes, quienes, sin temor a la coacción o la discriminación, pueden expresar sus puntos de vista y proponer cursos de acción.

Sin promover el relativismo moral, esta metodología identifica el problema o los múltiples problemas o conflictos dentro de una situación dada; estudia y propone diversas opciones y genera principios y postulados que pueden ser utilizados en escenarios futuros; se mantiene en continua dinámica entre el deber ser y el ser, entre el estudio de los ideales y el análisis del contexto, haciendo una prudente y mesurada interpretación de cada uno de los actores de la deliberación.

Este proceso dialógico es el que genera la preocupación por las otras personas, pues cumple la función de inclusión, buscando que la interpretación mutua refleje el verdadero sentir de cada uno de los participantes(9). Los conflictos abordados estudian el trasfondo filosófico y normativo moral de las relaciones interpersonales entre individuos y grupos, entre personas claramente identificadas e identificables

Sostiene Raúl Villarroel que con la bioética hermenéutica se puede realizar un aporte decisivo, al hacer surgir un nuevo modo de aproximación a los problemas fundamentales de la época y contribuir a la búsqueda de criterios de solución en la preparación de un nuevo arraigo para lo humano en los tiempos venideros; es decir, se busca sensibilizar o humanizar el avance científico, recuperando el valor de la vida humana y de su razón de ser, pero no en la teoría ni en la especulación, sino en la práctica y en los contextos locales o regionales, es decir, en la realidad cotidiana(8).

A partir de lo anterior, se concibe la bioética hermenéutica como una herramienta que sitúa en el centro de la argumentación la noción de interpretación dialógica esencial, la cual permite la expresión de una experiencia moral de diferentes tendencias y proyecciones, en una circularidad que no tiene tendencias ni deductivas ni inductivas, según lo afirma Adela Cortina en la conceptualización de la hermenéutica crítica(10).

Este contexto interdisciplinario ha tenido múltiples interpretaciones, puesto que, si bien no se busca la generación de consensos perfectos, sí se pretende que existan varias formas de abordaje de los conflictos (multidisciplinarmente). En este marco referencial, cada profesión o disciplina conserva y mantiene su conocimiento y perspectiva y lo comparte con los demás; interdisciplinarmente, es decir, trasladando métodos de una disciplina o profesión a otra, por ejemplo, aplicando 
nanotecnología al arte o, como en el caso de la bioética, en la que varias profesiones o disciplinas construyen conjuntamente una metodología deliberativa para el análisis de conflictos sociales.

\section{Dimensión bioética del abuso y el maltrato}

La aplicación de conceptos o determinaciones establecidas por entes o autoridades competentes administrativamente, hasta el momento no han logrado prevenir o modificar la conducta de maltrato y abuso en Colombia, a pesar de los avances. Existen, al parecer, grandes distancias entre los conceptos, las percepciones y las interpretaciones, las coyunturas y los procedimientos, pero realmente saber qué hay en el fondo es uno de los grandes retos de la actualidad. Ya decía Fernando Lolas que: "la urgente necesidad de reflexionar sobre los fines y medios en la actividad humana, que ya era problema filosófico en Grecia y por ende impregna todo el pensamiento occidental, no haya tomado suficientemente en cuenta la estrecha relación entre la retórica como ars bene dicendi y su paso al ars bene legendi, a la comprensión de los textos, pasando por la radical lingüisticidad de la experiencia humana que con justeza y elocuencia" (11).

Por tanto, el reto de la búsqueda de la comprensión de las acciones, textos y palabras en la relación intersubjetiva es fundamental; como no podemos comprender desde nuestra mirada o perspectiva, debemos intentarlo desde la perspectiva del otro o los otros. En este sentido, la bioética hermenéutica puede brindar una nueva posibilidad.

En la actualidad, las instituciones de salud enfrentan esta clase de problemas, como si cada caso fuera un fenómeno aislado, como una patología individual convertida en un "caso clínico" y, por lo tanto, como una anomalía independiente del contexto histórico, ético, político y social en el cual se presenta. El legislativo enfoca el problema desde el punto de vista sancionatorio y punitivo; otros sectores se marginan de las soluciones, remitiendo los casos al sector salud o a la justicia, como si el problema fuera exclusivamente sanitario o jurídico. El sector educativo está alejado de los procesos vivenciales y formativos en estos campos; porque en los procesos académicos no se le da suficiente importancia a la violencia in- trafamiliar como un componente de vulneración de derechos humanos, ni como parte integral del cuadro clínico ni del estudio de caso.

Este panorama es desalentador, si se tiene en cuenta que la Corte Constitucional colombiana, a través de sus sentencias, ha desarrollado un nuevo escenario jurídico y político en el cual los derechos de niños, niñas, adolescentes y mujeres deben estar en el rango de las garantías constitucionales, no solo de protección efectiva ante su vulneración sino de su materialización real; situación que es común a otros tantos programas de divulgación y prevención, nacionales o regionales sobre el tema.

Los estudios consultados permiten establecer que cuando se habla de maltrato y abuso infantil se está hablando de un problema de salud pública con evidentes conflictos éticos y morales(12), que debe ser enfrentado de manera articulada por los diferentes actores implicados. Por una parte, la sociedad debe impulsar la búsqueda de alternativas de cambio, comprensión y entendimiento de las diferentes situaciones que se presentan, rompiendo así progresivamente esquemas dicotómicos que han sido lesivos para la sociedad misma (víctima-victimario); por otra, el Estado, aval de los derechos de los niños, niñas, adolescentes y mujeres, y que tiene como función, a partir de propuestas y programas de salud pública intersectoriales, garantizar que las respuestas sean viables, deseables y acordes con las expectativas y condiciones contextuales de desarrollo comunitario, y que respondan a criterios éticos justificados. Finalmente, se requiere reflexiones públicas que incluyan tanto a los directos implicados como a los miembros de las instituciones que pueden aportar su experiencia, visión e iniciativas en la búsqueda de soluciones o modos de abordar los conflictos.

Ante esta situación, nuestro grupo de trabajo se apoya en un supuesto: necesitamos reconocer la violencia intrafamiliar y específicamente el maltrato y el abuso infantil como fenómenos complejos que se presentan en un marco sistemático. La violencia hacia los niños, niñas y adolescentes, en todas sus formas y en especial el abuso y maltrato infantil, pasa en muchos casos inadvertida como fenómeno social, porque es admisible o porque se tolera. Sin embargo, es importante tener en 
cuenta que, a pesar de que en los últimos años se ha presentado una tendencia a hacer visible tal violencia, no se ha eliminado aún el registro, y las cifras que se conocen no reflejan en realidad la dimensión del problema(13).

En este marco general, que parte del reconocimiento de las relaciones entre los diferentes miembros del grupo familiar, resulta necesario generar nuevas comprensiones y discursos sobre las familias y la realidad de las mismas, cuyas prácticas se modifican permanentemente como resultado de las nuevas configuraciones sociales, culturales y filiales. En esta propuesta, el maltrato y el abuso infantil se entienden en el marco de las relaciones asimétricas y de poder, inscritas en un contexto social y cultural que, dada su frecuencia, parece admitirlas, aunque, por otro lado, también las sanciona y condena(14).

\section{Perspectiva hermenéutica}

La hermenéutica es un método filosófico que parte del lenguaje simbólico para interpretar o significar las creencias y preferencias sociales o individuales del ser humano. No es un camino de dirección única, sino un cruce continuo de preguntas, réplicas y contestaciones. Esta interpretación implica un análisis conceptual de fondo; no puede ser algo meramente formal o arbitrario, sino el desarrollo de una gran capacidad de escuchar, entender y resignificar los contenidos expresados por el otro o los otros, sin desvirtuar ni la intencionalidad, ni los propósitos, ni el contexto en el que se da la situación conflictiva. Se requiere el desarrollo de una gran capacidad comunicativa para que la interpretación o el sentido interpretativo sean capaces de traducir lo que dice el contexto, el interlocutor o los interlocutores. Es un ejercicio que debe llevar implícito el reconocimiento del otro, su sentimiento y su sentido de ser, antes del propio.

Cuando se estudia la incidencia de las cuestiones morales en el campo de las ciencias de la vida, en el que coinciden medicina, biología, ciencias del medio ambiente y ciencias jurídicas y sociales, se encuentra que la bioética es: "El lugar común en el que se cruzan planteamientos éticos que cada vez resultan más difíciles de solventar. Porque las cuestiones que se nos plantean aparecen relacio- nadas con el sentido o el significado de gran parte de nuestras actividades o comportamientos, tanto en el terreno individual como colectivo. Los parámetros en los que se había pensado tanto la realización personal como la social han cambiado de tal forma que es precisa su reconsideración a la vista de la incidencia que tienen sobre ambas las nuevas tecnologías" (15).

Por ello, en algunas publicaciones se define a la bioética como un saber transdisciplinario e histórico-hermenéutico en permanente construcción, que se ocupa del cuidado responsable y solidario del comportamiento vital en la sociedad tecnocientífica desde tres puntos de vista: la vida, la calidad de vida y el sentido de la misma $(16,17)$, haciendo un ejercicio permanente de análisis y redefinición de términos, de ideas e imaginarios, para ubicar o clarificar cursos de acción. Es decir, se busca que sea aplicada, práctica y cercana a la realidad cotidiana, abordando temas como el sujeto y su identidad; la delimitación de lo humano y humanizador o, en su defecto, de lo inhumano y deshumanizador, y el aseguramiento técnicomoral — criterios de moralidad en una sociedad tecnológica - de un espacio limpio en el que se pueda llevar a cabo una vida digna de llamarse humana(15).

Este dejarse cuestionar desde fuera es lo que hace que el método de la transdisciplinariedad sea: "el método hermenéutico como modelo filosófico apto para el reencuentro de interpretaciones del que han de salir propuestas y/o criterios de enjuiciamiento de las actividades humanas. A este espacio reflexivo, que de metafórico se ha convertido en real, es al que hemos llamado 'lugar institucional"'.

Esto significa, entonces, que los escenarios dialógicos propiciados por la bioética son aquellos en los que yo considero al otro como si fuera yo mismo, buscando que en el reconocimiento intersubjetivo se promueva la justicia como acto humano, reflejo de su propia condición racional y sensible.

A nadie puede extrañar que, en este contexto, la hacer filosofía moral se convierta en urgente e ineludible; urgente, por cuanto la ética está urgida a "tener que dar cuenta" del sentido de las actividades que resultan determinantes para un 
significado de lo humano; e ineludible porque, en su búsqueda de razones, la filosofía moral no puede dejar de pensar, so pena de que la alternativa del aspecto visceral se erija en "juicio final" del sentido. A este respecto, todos reconocemos que las cuestiones que plantea la bioética constituyen uno de los lugares filosóficos por excelencia(15).

Las dicotomías éticas clásicas han de ser superadas en un "tercero" que constituya la verdad de ambas. Sin embargo, lo que es hoy patente en cualquier intento de fundamentar lo moral resulta diáfano en la ética aplicada: un solo modelo de ética es impotente para orientar las decisiones de los mundos político, económico, médico, ecológico, jurídico o, simplemente, de la convivencia ciudadana(10).

\section{Del niño como objeto al niño como sujeto de derechos}

Uno de los ejercicios difíciles durante el desarrollo de este proyecto fue el de la definición de "niño" o "niña”. En la práctica se notó que, dependiendo del sesgo, en la definición se expresan las posturas para una decisión, orientación o trato.

La racionalidad normativa está sustentada en una tradición y unos análisis positivos que, en el caso colombiano, apuntan a la búsqueda de evidencia para la definición de una conducta de carácter sancionatorio frente a un hecho que, socialmente, se califica como vulnerador de una norma.

La realidad social y cultural pone en evidencia una serie de posturas cambiantes a través del tiempo en el significado social de "niño" y "niña". La relación familiar, en especial en la construcción de las relaciones interpersonales o intersubjetivas, está mediada por los significados y conceptos de "persona", "ser humano", "ser social" y "ser familiar" que matiza la interpretación de roles de niños y nińas, los que, en un tiempo, tenían un cariz sin nigún valor, supuestamente para garantizar la participación neutral o inexistente de estos en las decisiones que se tomaban con su vida(18).

Pasando de ser propiedades de los padres a sujetos de protección y ahora sujetos de derechos, muy lejanos de los imaginarios y las realidades sociales, los niños y niñas siguen siendo considerados como "menores", "incapaces" e "inmaduros", pese a que se diga lo contrario en las normas. Tener en cuenta su individualidad y sus características contextuales de desarrollo, y su derecho a contar con condiciones de crecimiento, de acuerdo con sus ilusiones, perspectivas, actitudes, virtudes y demás, no se ha considerado aún como se hace con los adultos. Pareciera que el cambio en un dato cronológico pudiera dar al ser humano una connotación distinta para su consideración. Esto se evidencia claramente en la denominada inimputabilidad jurídica de los adolescentes menores de 18 años.

\section{La bioética y la salud pública}

La salud pública está enmarcada en normas constitucionales que expresan el resultado de evaluaciones sistemáticas y que, unidas a determinaciones de carácter político estratégico, implican también inversión económica con miras a impulsar cambios en las comunidades. Como políticas públicas, requieren de una serie de acciones que incrementen y que impliquen representatividad y legitimidad social, y por ende impacto en acciones que luego puedan ser analizadas mediante indicadores de avance o de retroceso.

La salud pública pretende asumir los problemas de salud en grupos o comunidades, un campo en el cual también interviene o participa la bioética, por lo que se pueden entrelazar o entrecruzar al menos sus intenciones u objetivos(19). Darras habla de dos perspectivas en la salud pública: una minimalista, que establece medidas de promoción y prevención en salud, en el marco de los denominados bienes públicos, y otra en que se involucran relaciones de poder. Pero, en términos generales, se pasa del tratamiento de la enfermedad a la planificación de la salud.

La bioética promueve la deliberación amplia, flexible, incluyente de todas las opciones y miradas. Esta salud pública existente tiende a definir opciones lineales, lo que deja por fuera muchas de las condiciones del contexto social que deberían ser tenidas en cuenta en la política pública. Por ello, creemos que debería existir una confluencia de aportes e intereses entre la salud pública y la bioética, para contribuir en la definición de la política pública. Si la salud pública amplia el con- 
cepto de salud a campos más allá de los clínicos y de los estudios de casos nosológicos, tomando en consideración las posturas morales de las personas que van a ejecutar o definir la política, podríamos tener nuevos elementos que, sin calificar, categorizar o definir aspectos puntuales del maltrato y del abuso, logren movilizar interiormente a los actores hacia nuevas formas de construir la relación o consideración intersubjetiva.

\section{Comentarios finales}

Este escrito es parte integral del proyecto de investigación "La bioética y la salud pública como referentes para la formulación intersectorial de políticas públicas orientadas a la prevención y manejo del maltrato y abuso infantil", dentro del cual quisimos asumir la bioética como un campo de conocimiento aún en construcción, caracterizado por su amplitud, para involucrar la diversidad conceptual tanto técnica como de valoración moral, es decir, como un elemento integrador de los aspectos médicos clínicos (como se concibió y aún se concibe en muchos ámbitos al abuso y al maltrato, al responder a elementos propios de la clínica y especialmente de la clínica nosológica); los aspectos jurídicos (cuando se considera en la práctica la existencia de seres de primera y segunda categoría: el menor de edad, el menor inmaduro, frente al mayor de edad, mayor competente); los aspectos culturales (la tendencia a dar mayor preeminencia al tener que al ser; la preponderancia y peso de la cultura religiosa que en muchas regiones es la mayor de todo el entorno), y los sentimientos, como parte integral de la racionalidad y de la reflexión, que en muchas ocasiones tienen más peso en las decisiones.
No pretendemos generar un instrumento para que la bioética pueda aportar a la formulación y mejor aplicación de la política pública en abuso y maltrato. Queremos construir, con las personas, nuevos conceptos, miradas, percepciones, vivencias que, in situ, generen una verdadera movilización moral, es decir, que a partir de la misma construcción hecha en los grupos de trabajo se genere una nueva conciencia hacia la existencia del otro o de los otros.

Teóricamente, nos aproximábamos a la ética del cuidado o de la protección, que la misma colectividad participante declaró en muchos momentos del proceso; sin embargo, el reto más grande asumido por el grupo lo constituyó la generación de las denominadas "categorías de encuentro", como escenarios de diálogo en los que no cabe la discriminación, valoración o jerarquización que profundice las distancias o los antagonismos entre los participantes.

Estas categorías de encuentro se trabajan en el momento del análisis, posterior al diálogo y la deliberación, como elementos generales de carácter formal, de manera que se investiga pero al mismo tiempo se dinamizan los grupos, disminuyendo las tensiones y propiciando el afloramiento de los sentimientos y la razón en el mismo espacio.

Esta nueva dinámica respondería a lo que Álvarez(20) define como uno de los grandes retos del estado democrático actual, que consiste en no solamente ser plural y laico, sino en delimitar los espacios de libertad y tolerancia para que cada ciudadano pueda ejercer la ética personal con la que se identifique de la mejor forma, sin querer imponerla al resto de sus conciudadanos. 


\section{Referencias}

1. Maldonado O, Cardozo de Martínez CA, et al. Maltrato y abuso infantil desde la relación bioética y salud pública. En: Violencia, Bioética y Salud Pública. Bogotá: Ministerio de Salud y Fondo de Población de las Naciones Unidas, UNPFA; 2010.

2. Cardozo de Martínez CA, et al. Implicaciones éticas, jurídicas y médicas de la sentencia C-355 de la Corte Constitucional: un avance para el ejercicio de los derechos humanos, sexuales y reproductivos de las colombianas. Bogotá: Ministerio de Protección Social y Universidad Nacional de Colombia; 2007.

3. Lolas F, Outomuro D. Bioética y Salud Pública. En: Lolas F, Quezada A, Rodríguez E, (eds.) Investigación en salud. Dimensión ética. Santiago de Chile: Centro Interdisciplinario de Estudios en Bioética, Universidad de Chile; 2006: 103-115.

4. Hoyos G. Estructuración del discurso bioético. Comunicación y lenguaje. En: Estatuto epistemológico de la bioética. México: Universidad Nacional Autónoma de México, Red de Bioética de la UNESCO; 2005.

5. Múnera L. La tragedia de lo público. (Conferencia) Bogotá: Universidad Nacional de Colombia; mayo de 2001.

6. Lolas F. Estudios de Bioética Social. Prioridades en salud y salud intercultural. Santiago de Chile: CIEB, Universidad de Chile; 2007.

7. Kottow M. Bioética pública: una propuesta. Bioética \& Debat 2011; 19(1): 61-76.

8. Villarroel R. Bioética hermenéutica Acta Bioéthica 2000; 6(1).

9. Cerrillo JA. El intermediario imposible. Algunas reflexiones en torno a epistemología y ética en la investigación cualitativa. Nómadas 2009; 24(4).

10. Cortina A. El estatuto de la ética aplicada. Hermenéutica crítica de las actividades humanas. Isegoría 1996; 13: 119-134.

11. Lolas F, Quezada A, Rodríguez E, (eds.) Investigación en salud. Dimensión ética. Santiago de Chile: Centro Interdisciplinario de Estudios en Bioética, Universidad de Chile; 2006.

12. Comité de Maltrato Infantil Sociedad Chilena de Pediatría. El maltrato infantil desde la bioética: el sistema de salud y su labor asistencial ante el maltrato infantil. ¿Qué hacer? Revista Chilena de Pediatría 2007; 78(Supl 1): 85-95.

13. Rivas F. La violencia como problema de salud pública en Colombia: otro campo para la bioética. Acta Bioethica 2000; 6(2).

14. Ovalle C. La bioética en la concepción, reivindicación y reconocimientos emergentes en los derechos humanos. Revista Colombiana de Bioética 2007; 2(2): 247-266.

15. González G. Bioética: saber y preocupación. En Junquera de Estéfani R, (dir.) Bioética y bioderecho. Reflexiones jurídicas ante los retos bioéticos. Granada; Ed. Comares; 2008: 5-38.

16. Rodríguez VH. Bioética, psicología y hermenéutica. Revista Latinoamericana de Bioética 2008; 8(1): 116-123.

17. Masiá Clavel JM. Revisión de la heteronomía en diálogo con Paul Ricouer. Revista de Filosofía Moral y Política 1992; 5: 17-27.

18. Gracia D. Bioética y pediatría. Revista Española de Pediatría 1997; 53(314): 99-106.

19. Darrás C. Bioética y Salud Pública. Al cruce de dos caminos. Acta Bioethica 2004;10(2): 227-233.

20. Álvarez Díaz J. Ética de mínimos y ética de máximos en el tema de los embriones criopreservados. Segunda Época 2012; $6(12)$.

Recibido: 27 de septiembre de 2013

Aceptado: 5 de noviembre de 2013 